\title{
Expression of the Apoptotic Proteins in Glioblastoma U87-MG Cell Line Treated by Botulinum Toxin
}

\author{
Mona Farhadi $^{*(\mathbb{D}}$, Samaneh Moniri ${ }^{1}$, Melikasadat Jameie ${ }^{2,3}{ }^{\circledR}$, Nasrin Hosseini $^{3^{\circledR}}$, Manasadat Jameie $^{2,3}{ }^{\circledR}$, \\ Seyed Behnamedin Jameie ${ }^{3,4}$
}

'Department of Microbiology, Karaj Branch, Islamic Azad University, Karaj, Iran

${ }^{2}$ Faculty of Medicine, Shahid Beheshti University of Medical Sciences, Tehran, Iran

${ }^{3}$ Neuroscience Research Center (NRC), Iran University of Medical Sciences, Tehran, Iran

${ }^{4}$ Department of Medical Basic Sciences, Faculty of Allied Medicine, Iran University of Medical

Sciences, Tehran, Iran

\begin{abstract}
Background: Glioblastoma multiforme (GBM) is the most type of brain malignancy in adults. Radical excision surgery, chemotherapy, and radiotherapy in some cases are still unsuccessful, and most patients with GBM die within 3 to 6 months following diagnosis. Botulinum toxin type A (BtxA) has cellular toxin effects and suppresses the cell division of certain types of cancer cell lines in vivo and in vitro study. The present study designed to evaluate the apoptotic effect of BtxA on the GBM cell line.

Methods: U87-MG GBM cell line cultured according to the routing protocols, divided into 2 groups including, trial (BtxA treated) and control groups. Cells of the trial group exposed to different doses of BtxA. The cell viability, cycle arrest, and pro-apoptotic proteins evaluated respectively by MTT assay, subG1, and Western blotting.

Results: MTT assay showed that the viability of the BtxA treated cells at doses of 1.45 Unit and other doses after 24 to 48 hours, significantly decreased $(P<0.001)$ compared to the control groups. Apoptosis percentage of the subG1 test also indicated that 1.45 unit dose significantly increased in the cells exposed to BtxA compared to the control group in 24 hours. The expression of P53 and caspase 3 proteins indicated a significant increase.

Conclusion: BtxA induces apoptosis in U87-MG cell line via p53 and caspase 3 pathways and could have clinical applications. In vivo studies need to confirm the clinical application of the present findings.

Keywords: Glioblastoma multiforme; U87-MG cell line; Botulinum toxin type A; P53; Caspase 3.
\end{abstract}

\author{
*Correspondence to \\ Mona Farhadi, Assistant \\ Professor, Department of \\ Microbiology, Karaj Branch, \\ Islamic Azad University, Karaj, \\ Iran \\ E-mail: mona.farhadi@kiau. \\ ac.ir \& mona.farhadi3712@ \\ gmail.com
}

Published online June 21, 2020

Citation: Farhadi M, Moniri S, Jameie M, Hosseini N, Jameie M, Behnamedin Jameie SB.Expression of the Apoptotic Proteins in Glioblastoma U87-MG Cell Line Treated by Botulinum Toxin. Clin Neurosci J. 2020;7(3):132-137. doi:10.34172/icnj.2020.14.

\section{Introduction}

Glioblastoma multiforme (GBM) is the most common and malignant type of brain tumor that has a mean patient survival of about 14 months despite the surgery, chemotherapy, and radiotherapy. Routine therapeutic procedures, including invasive surgery, chemotherapy, and radiation therapy, are still unsuccessful. GBM is highly metastatic, and like most other nervous tissue tumors is not sensitive to chemotherapy. ${ }^{1}$ Other factors, such as the disruption of the blood-brain barrier, the loss of well-defined boundary between tumor cells and healthy nervous tissues, and intolerance of healthy cells against radiation, lead to the failure of standard therapeutic methods. ${ }^{2}$ Surgery, chemotherapy, and radiotherapy are not still able to increase the rate of patient survival. Therefore, the treatment of central nervous system (CNS) tumors aims to establish good living conditions by reducing each therapy's neurological side effects. However, disappointing results have led to the use of new and combined therapies in clinical and experimental research. Botulinum toxin type A (BtxA) is a protein produced by bacterium Clostridium. BtxA is lethal but now consider as an active treatment agent. BtxA is used to treat muscular spasm, facial paralysis, chronic lumbar pain, specific symptoms of stroke, brain damage, and neuromuscular disorders. ${ }^{3}$ Rajkumar et al in 2005 reported the clinical efficacy of intravesical injection of BtxA on detrusor muscle of urinary bladder in patients with urinary bladder dysfunction. ${ }^{4}$ They showed that BtxA decreased signs and symptoms of the disease

(C) 2020 The Author(s). This is an open access article distributed under the terms of the Creative Commons Attribution License (http:// creativecommons.org/licenses/by/4.0/), which permits unrestricted use, distribution, and reproduction in any medium, provided the original work is properly cited. 
without any adverse effects. ${ }^{4}$ Darwish et al in 2014 showed that BtxA might lead to undesirable histopathological effects in testicular tissue of adult rats treated with BtxA that might lead to infertility. ${ }^{5}$ There are also other studies indicating that BtxA is a safe and effective treatment in a dose-dependent manner. ${ }^{6,7}$ Many studies focus on the in vitro investigation of BtxA.

Karsenty et al, in 2009, showed induction of apoptosis of the human prostate cancer LNCaP and PC-3 cell lines by BtxA in a dose-dependent manner. ${ }^{8}$ In 2013 Bandala et al conducted a study to evaluate the apoptotic effect of BtxA on T47D breast cancer cells. According to their results, BtxA caused significant cytotoxic and apoptotic effects on T47D cells. ${ }^{9}$ How BtxA induces apoptosis is still unknown. However, it seems that BtxA may act throughout the intrinsic pathway of apoptosis involving p53 and caspase 3 proteins. The p53 protein involves stopping cell cycles and the onset of apoptosis in response to DNA damage. It also stimulates the transcription of several genes that stop cell cycles and initiates apoptosis. The cessation of the cell cycle occurs by p53 at the end of the G1 stage and has generated by a transcriptional p53-dependent inhibitor of CDK (cyclin-dependent kinase), i.e., p21. ${ }^{10}$ Caspases are family proteins that are crucial mediators of apoptosis. Among them, caspase 3 is a frequently activated death protease, catalyzing the specific cleavage of many vital cellular proteins. Caspase 3 is essential for healthy brain development and is essential for apoptotic scenarios in remarkable tissue. Caspase-3 is also required for some typical hallmarks of apoptosis and is essential for apoptotic chromatin condensation and DNA fragmentation. To our knowledge, there is a lack of enough evidence for the effects of BtxA on the GBM cell line. Thus, regarding the anti-proliferative and apoptotic role of BtxA, the present research designed in order to study the apoptotic effects of BtxA on the GBM cell line with particular focus on hallmark role of $\mathrm{p} 53$ and caspase 3.

\section{Materials and Methods \\ Reagents and Chemicals}

DMEM, FBS (Gibco), and DMSO purchased from Sigma Aldrich. Botox (Canitox as the most common brand in Iran), p53 (1C12) and caspase 3 (9662s) Mouse mAb were purchased from Cell Signaling Technology Inc. Propidium iodide was purchased from Abcam.

\section{Cell Culture}

The U87MG glioblastoma cell line has purchased from the Tehran Pasteur Institute. Cells cultured in DMEM medium containing $10 \%$ FBS with a cell density of $10^{4}$ cells $/ \mathrm{cm}^{2}$, and was then placed in incubators with $5 \%$ of $\mathrm{CO}_{2}$ and $98 \%$ of humidity at $37^{\circ} \mathrm{C}$ and stored up to 10 passages.

\section{Microscopic Evaluated}

The cells before and after treatment have evaluated by invert microscopy.

\section{Viability assay}

Two groups of cells, including treated cells with different concentrations of BtxA and control with an approximate cell number of $1 \times 10^{4}$ cultured in 96-well plates studied in this test. The 96-well plate was moved into the incubator for 24 hours, following adhesion of cells, BtxA treatment for 24 and 48 hours with different doses of $0.75,0.85,0.95$, $1.05,1.15,1.25 .1 .35,1.45,1.65,1.85,2.05$ units has done. All treatment steps performed on ice and MTT solution was added to each well incubated at $37^{\circ} \mathrm{C}$ for $2-4$ hours. The obtained optical absorption read by EllSA reader at $570 \mathrm{~nm}$.

\section{SubG1 Assay}

The control and BtxA treated cells observed for the cell cycle analysis. In this method, cells were evaluated after fixation and staining with PI through the flow cytometry. PI color is capable of binding to DNA and RNA in fixed cells, and thus RNase is used for stain cells so that only the DNA content of cells could detect and measured the loss of RNA.

For subG1 assay, the cells were cultured in a 12-well plate and divided into 2 groups of control, and BtxA treated cells. Trail cells exposed to the $1.45 \mathrm{U}$ and $1.65 \mathrm{U}$ doses of BtxA for 24 and 48 hours. Supernatant removed, and cells washed with PBS and 70\% alcohol. Cells have kept at $-20^{\circ} \mathrm{C}$ for 24 hours. RNase and PI added at a concentration of $1 \mathrm{mg} / \mathrm{mL}$, incubated for 30 minutes at ambient temperature in a dark place; they were read by flow cytometry after 30 minutes.

\section{Western Blotting for Caspase 3 and p53 Proteins}

The protein was extracted from the cell and placed on the SDS-PAGE gel. The SDS PAGE gel used to purify proteins of U87MG cells. Thirty micrograms protein was separated on the gel-protein samples and transferred to the polyvinylidene difluoride (PVDF) membrane, and eventually, protein samples, which revealed on the PVDF membrane, were detected using the ECL kit. After blocking with buffer containing 3\% of ECL Advance in PBS/Tween $0.3 \%$ for 1.5 hours at room temperature, the membrane probed with specific polyclonal antibodies (Abcam) diluted in blocking solution (Caspase3 1:7500, P53 1:7500) antibodies were added to the membrane 1 hour at room temperature. Then, a secondary antibody added onto the membrane. After the incubation time, the secondary antibodies were removed and washed by a trisbuffered saline (TBS) buffer for 3 to 10 minutes.

\section{Statistics}

All experiments were repeated at least 3 times. The data 
were presented as means \pm standard deviation (SD). The statistical analysis was performed using KolmogorovSmirnov to check the normal distribution of data; and Tukey test to check the equality of variances. The one-way ANOVA was used for comparison.

\section{Results}

Microscopic study showed the BtxA treated cells were detached and changed in shape, granulation compared to the cells of the control group. These features showed early signs of apoptosis in BtxA treated cells (Figure 1).

Viability test showed a significant difference between the percentage of live cells in the control group compared to BtxA treated cells at doses of 1.45, 1.65, 1.85 and 2.05 units $(P<0.001)$ after 24 and 48 hours in a dose-dependent manner. There was a non-significant difference between 24 and 48 hours BtxA treated groups, the number of live cells in BtxA treated within 24 hours was less than the live cells within 48 hours of treating (Figure 2).

For the subG1 assay, the BtxA treated cells at doses of 1.45 and 1.65 units within 24 hours, which showed meaningful results in MTT assay, were used.

Treating with doses of 1.45 and 1.65 Units within 24 hours showed $56.58 \%$ and $83.63 \%$ of dead cells compared to the control group, respectively (Figure 3 ).

Figure 4 shows the comparison of apoptosis between
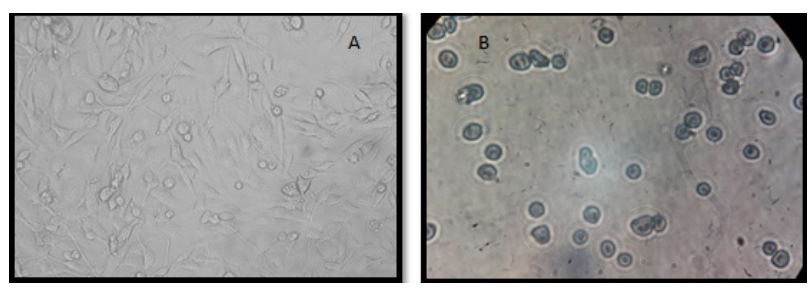

Figure 1. Micrograph of control cells group (A) and U87-MG cells BtxA treated groups $(B)$ after 24 hours $(\times 100)$.

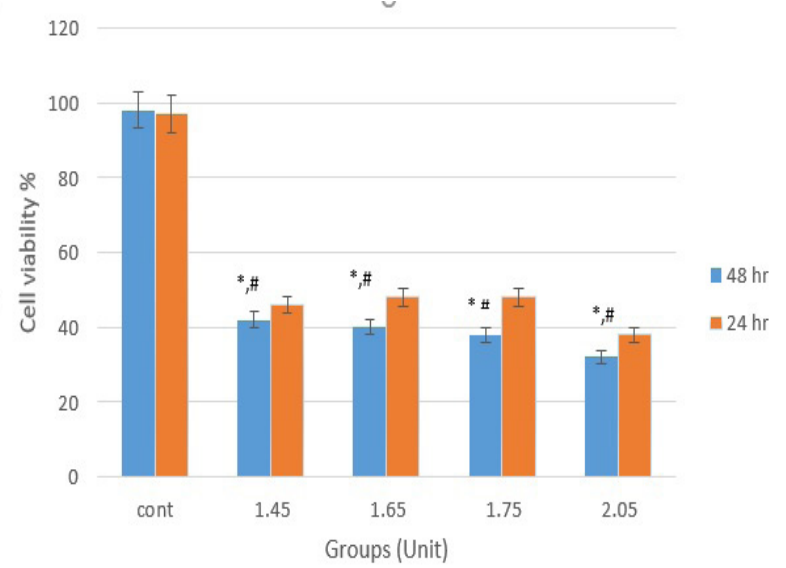

Figure 2. MTT assay: The Percentages of Live Cells in Control and BtxA Treated Groups After $24 \mathrm{~h}$ and $48 \mathrm{~h}$. Mean \pm SD, $* P<0.00148 \mathrm{hr}$ compared to the control at the same time. $\# P<0.00148 \mathrm{hr}$ compare to $24 \mathrm{hr}$.
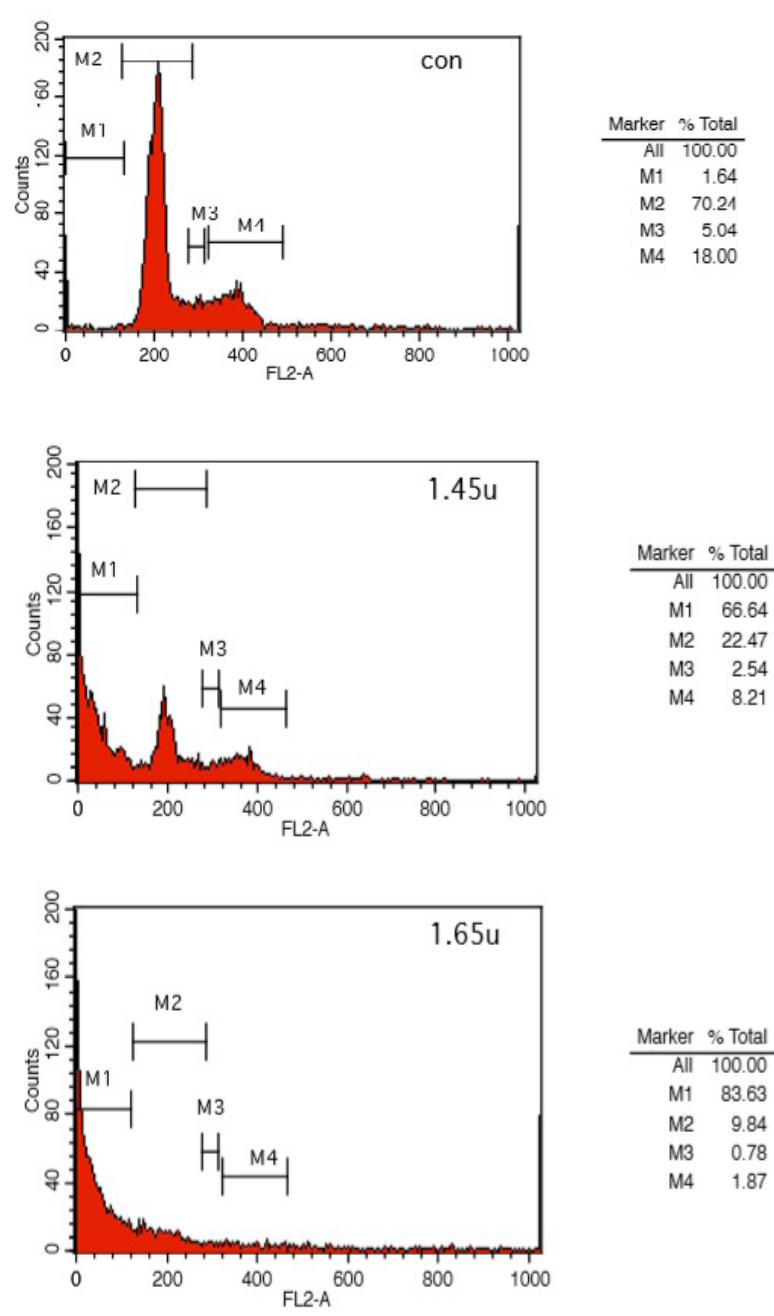

Figure 3. SubG1 Assay of Botox Treatment and Control Groups at 1.45 and 1.65 Unit Dose After 24 Hours.

24 and 48 hours of BtxA treatment at a dose of 1.45. Accordingly, there was a significant difference between the number of apoptotic cells in the BtxA treated group at 1.45 Units within 24 hours compared to the same dose within 48 hours of BtxA treating $(P<0.05)$.

Western blotting results showed a significant increase in caspase 3 and p53 in BtxA treated group at the dose of 1.45 units within 24 hours compared to the control group $(P<0.001)$.

\section{Discussion}

Radical invasive surgery following by radiation and chemotherapy still is the first clinical decision to treat patients with GBM. ${ }^{11}$ Although this type of treatment is used frequently for most cases of cancers, unfortunately, there is the loss of enough success since most brain tumors are recurrent and not sensitive effectively to currently chemicals agents. ${ }^{12}$ Due to difficulties in surgery of anatomical position of the tumors, not enough sensitivity of tumor cells to chemotherapy and radiation therapy, 


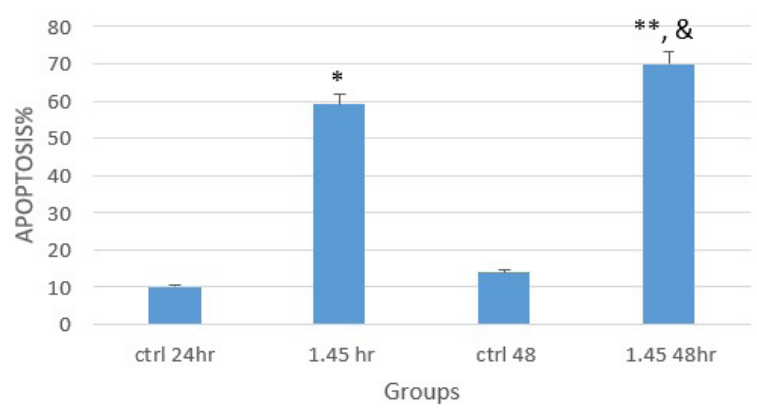

Figure 4. The Percentage of Apoptotic Cells by SubG1 Assay. mean $\pm \mathrm{SD}, * P<0.05,{ }^{* *} P<0.001$ significant differences compared to control. \& $P<0.05$ significant differences Compared between groups $P<0.05$

recently, researchers are thinking of adjuvant therapies. ${ }^{13}$ Since the Food and Drug Administration (FDA) approved the BtxA, it has widely applied in clinical practice. BtxA is known to act at nerve endings by blocking the exocytosis of various neurotransmitters through the endopeptidase activity of its light chain that cleaves SNAP-25. A protein belonging to the SNARE (soluble N-ethyl maleimidesensitive factor attachment protein receptor) complex known to regulate the fusion of presynaptic vesicles to the plasma membrane. ${ }^{8}$ As our data showed, BtxA has the potential to induce apoptosis in U87-GM GBM cell line in the dose and time-dependent manner. Some other similar researches are using BtxA for the treatment of a certain type of tumor cells. Treating T47D breast cancer cell line and MCF10A normal cell line by BtxA reported by Bandala et al in 2013. They showed that BtxA has cytotoxic effects on these cells; their results showed that the effectiveness of BtxA on T47D cells is more than the effects of BtxA treating of MCF10A. The dosing unit that they used for BtxA was 5.3 units within 24 hours of exposure time. They also claimed that the BtxA induces apoptosis via activation of caspases 3 and 7. ${ }^{9}$ Their results are close to our findings on the GBM cell line with some differences. Despite the similarity in exposing and effective time of BtxA, the effective dose of our research (1.45 unit) was different from their dose (5.3 unit) that might be due to the sensitivity of the cell lines. Our data confirmed the role of caspase 3 in apoptosis induction. In another study, Karsenty et al showed the effectiveness of BtxA on the human LNCaP prostate cancer cell line. They showed that the BtxA induces apoptosis and prevents the growth of prostate cancer cells in vitro and in vivo. ${ }^{9}$ They showed the role of synaptic vesicles (SV) proteins such as SV2 membrane receptor for the act of BtxA on LNCap cell line and hypothesized that the presence of SV2 in LNCaP might allow the BtxA light chain to enter the cells and, block exocytosis of paracrine or autocrine factors involved in cell proliferation. There are some controversies regarding the apoptotic effect of BtxA. Although some researchers have pointed to the apoptosis induction role of BtxA, other researchers have shown that BtxA could protect against apoptosis. Shi et al, in 2019, showed the antiapoptotic effects of BtxA on human dermal microvascular endothelial cells. According to them, BtxA prevents cytochrome C release and by decreasing the expression of caspase 3 , inhibits apoptosis. ${ }^{13}$ Their results are in contrast with the findings of this study, and we guess the type of the cell line might consider as the factor that influences the results, or it is also possible that the BtxA goes by different pathways in various cells to inhibit or induce apoptosis. We also showed an increase in the expression of p53 and caspase 3 in BtxA treated cells. The p53 gene is the most-widely known tumor suppressor gene and plays a major role in inducing apoptosis ${ }^{14}$ also, caspase- 3 is essential for apoptotic chromatin condensation and DNA fragmentation. Regarding Karsenty et al explanation, we hypothesized that there might be some mechanisms for the effect of BtxA on the U87-GM cell line. We think that the activation of the p53 and caspase 3 might be due to the BtxA effect on membrane receptors of the GBM cell line.
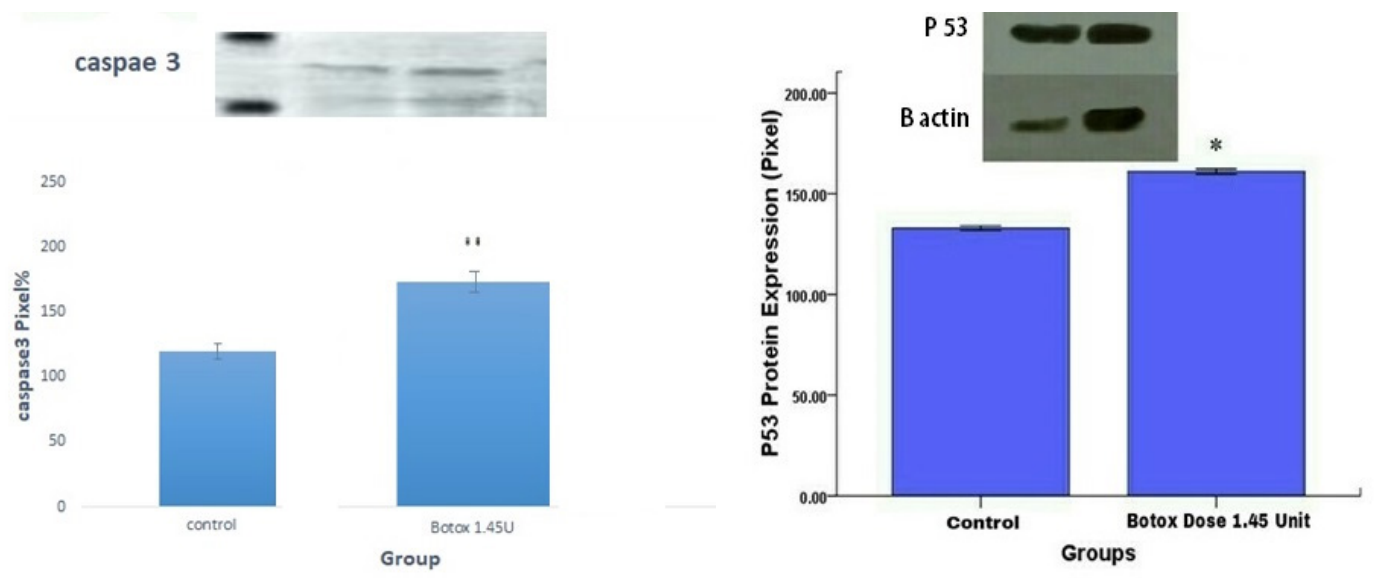

Figure 5. The Expression of Caspase 3 \& P53 Protein by Western Blotting Between the Control and 1.45 BtxA group. Mean \pm SD, $* P<0.05,{ }^{* *} P<0.001$. 
Zhang et al mentioned the details of the p53 pathway in GBM. They emphasized the deregulation of the p53-ARFMDM2 pathway at $84 \%$ of GBM patients and $94 \%$ of the GBM cell line. According to their results, the deregulated p53 pathway component has implicated in GBM cell line invasion, migration, proliferation, initiation of apoptosis, and cancer cells. ${ }^{14}$ Analysis and the clinical importance of the expression of receptors in different grade (I-IV) of GBM reported by Hu et al. ${ }^{15}$ The expression of the SV proteins family of GBM reported by de Groot et al. ${ }^{16}$ They showed the expression of SV2 in GBM of grade II patients. They also claimed that the expression of SV2 might show a better prognosis for these patients. From a clinical point of view about $\mathrm{p} 53$, Jin et al in 2016 reported the prognostic importance of $\mathrm{p} 53$ in patients with GBM. They concluded that p53 immunohistochemical expression might have effective usefulness in predicting the prognosis in patients with glioma. ${ }^{17}$ So BtxA and other adjuvant therapy with the capability of increasing the p53 expression could be useful to the treatment of this type of malignancy.

Regarding the expression of caspase 3, we showed that BtxA increased the level of its expression significantly in the GBM cell line. Tirapelli et al in 2010 reported the low expression of caspase 3 in samples of patients with GBM that in turn shows the resistance of GBM cell to apoptosis. ${ }^{18}$ So any treatment increased the expression of caspase 3 might be clinically useful. In addition to the novelty of treating GBM cell line by BtxA that first reported, we also showed the expression of p53 and caspase 3 with more focus on p53, which is very close to recent findings role of the $\mathrm{p} 53$ pathway in treatment and prognosis of patients with GBM.

\section{Conclusion}

We reported the first observation of growth inhibition and apoptosis in the U87-GM GBM cell line by BtxA in vitro. Based on our findings, BtxA has the potential to suppress U87-MG GBM cells and inducing apoptosis via activation of the intrinsic apoptotic pathway. BtxA might use clinically for human GBM tumors. However, it needs further investigations. Whether BtxA could use along with other routine therapeutic procedures for patients with GBM is also a matter of research and question.

\section{Conflict of Interest}

The authors declare no conflict of interest.

\section{Acknowledgments}

The authors are deeply grateful to colleagues and laboratory staff of NRC/IUMS for their sincere collaboration and also Vice-Chancellor Of Research and Technology.

\section{Authors' Contribution}

$M F, S M$, and $M J$ involved in experiments and wrote the paper draft.
$\mathrm{NH}$ and $\mathrm{MJ}$ collected the data. MJ edited the paper, and all the authors read and approved the final manuscript.

\section{Funding/Support}

The present research was supported by the Neuroscience Research Center (NRC) of the Iran University of Medical Science and Islamic Azad University.

\section{Ethical Statement}

The study was conducted under the principles of the Declaration of Ethical Committee of Iran University of Medical Sciences for Experimental and Cellular research and approved by the Ethical Committee of NRC/IUMS.

\section{References}

1. Hengartner MO. The biochemistry of apoptosis. Nature. 2000;407(6805):770-6. doi: 10.1038/35037710.

2. Cloughesy TF, Yoshimoto K, Nghiemphu P, Brown K, Dang J, Zhu S, et al. Antitumor activity of rapamycin in a Phase I trial for patients with recurrent PTEN-deficient glioblastoma. PLoS Med. 2008;5(1):e8. doi: 10.1371/journal.pmed.0050008.

3. Blachon S, Demeret C. The regulatory E2 proteins of human genital papillomaviruses are pro-apoptotic. Biochimie. 2003;85(8):813-9. doi: 10.1016/j.biochi.2003.09.008.

4. Rajkumar GN, Small DR, Mustafa AW, Conn G. A prospective study to evaluate the safety, tolerability, efficacy and durability of response of intravesical injection of botulinum toxin type A into detrusor muscle in patients with refractory idiopathic detrusor overactivity. BJU Int. 2005;96(6):848-52. doi: 10.1111/j.1464-410X.2005.05725.x.

5. Darwish HA, Arab HH, Abdelsalam RM. Chrysin alleviates testicular dysfunction in adjuvant arthritic rats via suppression of inflammation and apoptosis: comparison with celecoxib. Toxicol Appl Pharmacol. 2014;279(2):129-40. doi: 10.1016/j. taap.2014.05.018.

6. Radwan MM, Ramdan K, Abu-Azab I, Abu-Zidan FM. Botulinum toxin treatment for anal fissure. Afr Health Sci. 2007;7(1):14-7. doi: 10.5555/afhs.2007.7.1.14.

7. Abramovitz A, Goyeneche C, Cánchica A, Burgues T, Sucupira E. Botulinum toxin application in the facial muscles for the treatment of stuttering. Journal of Laryngology and Voice. 2017;7(1):14-7. doi: 10.4103/jlv.JLV_6_17.

8. Karsenty G, Rocha J, Chevalier S, Scarlata E, Andrieu C, Zouanat FZ, et al. Botulinum toxin type A inhibits the growth of LNCaP human prostate cancer cells in vitro and in vivo. Prostate. 2009;69(11):1143-50. doi: 10.1002/pros.20958.

9. Bandala C, Perez-Santos JL, Lara-Padilla E, Delgado Lopez G, Anaya-Ruiz M. Effect of botulinum toxin A on proliferation and apoptosis in the T47D breast cancer cell line. Asian Pac J Cancer Prev. 2013;14(2):891-4. doi: 10.7314/ apjcp.2013.14.2.891.

10. Bieging KT, Mello SS, Attardi LD. Unravelling mechanisms of p53-mediated tumour suppression. Nat Rev Cancer. 2014;14(5):359-70. doi: 10.1038/nrc3711.

11. Ghafoor A, Jemal A, Cokkinides V, Cardinez C, Murray T, Samuels A, et al. Cancer statistics for African Americans. CA Cancer J Clin. 2002;52(6):326-41. doi: 10.3322/ canjclin.52.6.326.

12. Galanis E, Buckner J. Chemotherapy for high-grade gliomas. $\mathrm{Br}$ J Cancer. 2000;82(8):1371-80. doi: 10.1054/bjoc.1999.1075.

13. Shi Y, Lin H, Cao J, Cui C. Botulinum Toxin Type A Attenuates Apoptosis in Human Dermal Microvascular Endothelial 
Cells Exposed to an In Vitro Model of Ischemia/Reperfusion Injury. Transplant Proc. 2019;51(3):966-971. doi:10.1016/j. transproceed.2018.11.006

14. Zhang Y, Dube C, Gibert M Jr, Cruickshanks N, Wang B, Coughlan M, et al. The p53 Pathway in Glioblastoma. Cancers (Basel). 2018;10(9). doi: 10.3390/cancers10090297.

15. Hu G, Wei B, Wang L, Wang L, Kong D, Jin Y, et al. Analysis of gene expression profiles associated with glioma progression. Mol Med Rep. 2015;12(2):1884-90. doi: 10.3892/ mmr.2015.3583.

16. de Groot M, Aronica E, Heimans JJ, Reijneveld JC. Synaptic vesicle protein $2 \mathrm{~A}$ predicts response to levetiracetam in patients with glioma. Neurology. 2011;77(6):532-9. doi: 10.1212/WNL.0b013e318228c110.

17. Jin $Y$, Xiao W, Song T, Feng G, Dai Z. Expression and prognostic significance of p53 in glioma patients: a metaanalysis. Neurochem Res. 2016;41(7):1723-31. doi: 10.1007/ s11064-016-1888-y.

18. Tirapelli LF, Bolini PH, Tirapelli DP, Peria FM, Becker AN, Saggioro FP, et al. Caspase-3 and Bcl-2 expression in glioblastoma: an immunohistochemical study. Arq Neuropsiquiatr. 2010;68(4):603-7. doi: 10.1590/s0004$282 \times 2010000400023$. 